\title{
Transtorno bipolar pediátrico e TDAH comórbidos: relato de caso
}

Pediatric bipolar disorder and comorbid ADHD: case report

Trastorno bipolar pediátrico y TDAH comórbido: reporte de caso

Daniel Cardoso de Bastos

- https://orcid.org/0000-0003-4006-9451

Lorena Lourenço da Cruz de Carvalho - https://orcid.org/0000-00026387-4982

Vitor Melo Zibordi - https://orcid.org/0000-0001-7969-2174

Higor Bermudes Nascimento - https://orcid.org/0000-0002-1630-1073

Sonia Maria Motta Palma - https://orcid.org/0000-0003-3152-4758

\section{RESUMO:}

Introdução: O Transtorno de déficit de atenção/hiperatividade e o Transtorno Bipolar Pediátrico são patologias psiquiátricas de curso crônico, com apresentação já na infância e adolescência, persistindo na idade adulta. A comorbidade entre esses transtornos costuma ocorrer e é comumente associada a outras condições médicas e psiquiátricas, levando a piora do quadro, impactando negativamente em aspectos da vida do paciente. Esse relato descreve o caso de um paciente do sexo masculino, 17 anos, diagnosticado com TDAH e TB em comorbidade na infância. Objetivo: Além do relato de caso, este artigo descreve as características do TDAH e TB na infância, segundo os critérios clínicos e diretrizes diagnosticas atuais a fim de mostrar aos profissionais da saúde a dificuldade e importância do diagnóstico dessas patologias e o seguimento terapêutico em caso de comorbidade. Métodos: Os dados foram obtidos por meio de análise de prontuário e revisão literária. Conclusão: É necessário a realização de novas pesquisas e debates voltados a esse tema, principalmente com enfoque em aspectos que não se sobreponham, a fim de facilitar o diagnóstico comórbido e/ou diferencial do TB e TDAH na infância e, consequentemente, minimizar seus prejuízos ao indivíduo. 
Palavras chave: transtorno bipolar pediátrico, transtorno de déficit de atenção e hiperatividade, comorbidade, diagnóstico

\section{ABSTRACT:}

Introduction: Attention Deficit/Hyperactivity Disorder and Pediatric Bipolar Disorder are psychiatric pathologies with a chronic course, presenting as early as childhood and adolescence, and persisting into adulthood. Comorbidity between these disorders usually occurs and is commonly associated with other medical and psychiatric conditions, leading to worsening of the condition, negatively impacting aspects of the patient's life. This report describes the case of a 17-year-old male patient diagnosed with ADHD and TBP in comorbidity. Objective: In addition to the case report, this article describes the characteristics of ADHD and TB in childhood, according to clinical criteria and current diagnostic guidelines, in order to show health professionals, the difficulty and importance of diagnosing these pathologies and the therapeutic follow-up in case of comorbidity. Methods: Data were obtained through analysis of medical records and literature review. Conclusion: It is necessary to carry out further research and debates on this topic, mainly focusing on aspects that do not overlap, in order to facilitate the comorbid and/or differential diagnosis of TB and ADHD in childhood and, consequently, minimize their damage to the individual.

Keywords: pediatric bipolar disorder, attention deficit hyperactivity disorder, comorbidity, diagnosis

\section{RESUMEN:}

Introducción: El Trastorno por Déficit de Atención / Hiperactividad y el Trastorno Bipolar Pediátrico son patologías psiquiátricas con un curso crónico, que se presentan desde la niñez y la adolescencia y persisten hasta la edad adulta. La comorbilidad entre estos trastornos suele ocurrir y se asocia comúnmente con otras afecciones médicas y psiquiátricas, lo que lleva a un empeoramiento de la afección, impactando negativamente en aspectos de la vida del paciente. Este informe describe el caso de un paciente masculino de 17 años diagnosticado de TDAH y TBP en comorbilidad. Objetivo: Además del caso clínico, este artículo describe las características del TDAH y la TB en la infancia, según criterios clínicos y 
guías diagnósticas vigentes, con el fin de mostrar a los profesionales de la salud la dificultad e importancia del diagnóstico de estas patologías y el seguimiento terapéutico. en caso de comorbilidad. Métodos: Los datos se obtuvieron mediante análisis de historias clínicas y revisión de la literatura. Conclusión: Es necesario realizar más investigaciones y debates sobre este tema, centrándose principalmente en aspectos que no se superponen, con el fin de facilitar el diagnóstico comórbido y / o diferencial de TB y TDAH en la infancia y, en consecuencia, minimizar su daño a el individuo.

Palabras clave: trastorno bipolar pediátrico, trastorno por déficit de atención con hiperactividad, comorbilidad, diagnóstico

Como citar: Bastos D.C., Carvalho L.L.C., Zibordi V.M., Nascimento H.B, Palma S.M.M, - Transtorno bipolar pediátrico e TDAH comórbidos: relato de caso. Debates em Psiquiatria, Rio de Janeiro, 2021; 11:1-13.

https://doi.org/10.25118/2763-9037.2021.v11.242

Conflito de interesses: declaram não haver

Fonte de financiamento: declaram não haver

Parecer CEP: não se aplica

Recebido em: 29/10/2021

Aprovado em: 08/11/2021

Publicado em: 12/11/2021

\section{Introdução}

O Transtorno de déficit de atenção/hiperatividade (TDAH) e o Transtorno Bipolar Pediátrico são patologias psiquiátricas de curso crônico, com apresentação já na infância e adolescência e persistência na idade adulta, ocasionando grandes impactos nos aspectos sociais, educacionais e familiares. A comorbidade entre esses transtornos é comumente associada a outras condições médicas e psiquiátricas, levando a piora do quadro, com aumento da impulsividade, sintomas psicóticos, necessidade de hospitalização e piora no desempenho escolar. [1, 2$]$

O TDAH é um transtorno do neurodesenvolvimento, iniciando-se precocemente (antes dos 12 anos) e definido por sintomas persistentes de desatenção e / ou hiperatividade e impulsividade que afetam no 
funcionamento e desenvolvimento da criança, por no mínimo 6 meses e em pelo menos dois ambientes, por exemplo, em casa e na escola. [3] Crianças e adolescentes com TDAH podem apresentar irritabilidade e descontrole de impulsos, porém, tendem a ser mais pontuais quando associados ao transtorno, ou seja, frente a uma frustração ou contrariedade e após um tempo razoavelmente curto, desaparecem. Normalmente, não há alteração de humor permanente ou mesmo em períodos longos nos indivíduos com TDAH. Na verdade, uma parcela grande das crianças com o transtorno e sem outras comorbidades é descrita como alegre, despreocupada e de fácil socialização. Eventualmente, quando o mesmo se associa ao transtorno de oposição desafiante, a diferenciação entre a oposição constante a regras e irritabilidade pode ser mais difícil. [4]

O Transtorno Bipolar (TB) é um transtorno mental crônico e incapacitante, caracterizado por alterações de humor (mania/hipomania e depressão). [므, 6] A idade média do primeiro episódio é no final da adolescência ou início da fase adulta, entretanto seu início na infância é possível. Em crianças e adolescentes, pode se manifestar de forma diferente do padrão descrito na idade adulta, não cumprindo totalmente os critérios diagnósticos. [ $\underline{3}$ ] A irritabilidade associada a significativo descontrole de impulsos é a alteração de humor mais comum em crianças e adolescentes com TB, já que as mesmas tendem a não apresentar apenas humor eufórico como nos quadros clássicos do adulto.[4] Além disso, a autoestima inflada e a sensação de grandiosidade presentes nos adultos, pode se apresentar de forma diferente em crianças, supervalorizando suas capacidades, acreditando ser melhor e mais inteligente do que outras crianças. Já no período depressivo, elas podem apresentar humor irritável ao invés de triste. [ $\underline{3}]$

Ambos os transtornos são frequentemente não diagnosticados ou mal diagnosticados e, às vezes, superdiagnosticados, levando a altas taxas de morbidade e incapacidade. A diferenciação dessas condições é baseada em suas características clínicas, comorbidade, história familiar psiquiátrica, curso da doença e resposta ao tratamento.[1] Além disso, a comorbidade entre o TDAH e TB foi negligenciada por muito tempo, pois não se acreditava que havia continuidade do TDAH na idade adulta e que o TB poderia iniciar na infância. A existência de altas taxas de comorbidade entre ambos os transtornos é outro desafio para o diagnóstico. De 57 a $98 \%$ das crianças com TB também atendem aos critérios para o TDAH. De 
11 a 23\% das crianças com TDAH também atendem aos critérios de diagnóstico do TB. []]

Nas últimas décadas, o Transtorno bipolar pediátrico assim como sua sobreposição sintomática com o TDAH tornou-se uma nova questão para debates entre pesquisadores devido o surgimento de uma quantidade considerável de relatos de casos. A partir disso, avanços importantes vêm sendo descobertos nesse âmbito. []]

O objetivo deste relato de caso é descrever a comorbidade entre TDAH e Transtorno Bipolar na infância segundo critérios clínicos e diretrizes diagnósticas atuais (DSM-5), para que possamos mostrar aos profissionais da saúde a dificuldade e importância do diagnóstico dessas patologias e o seguimento terapêutico em caso de comorbidade. O paciente e seus familiares autorizaram a publicação desse relato e nomes não foram citados para proteger suas identidades sem prejuízo à veracidade do caso clínico.

\section{Relato de caso}

N.S.M., 17 anos, masculino, iniciou acompanhamento no ambulatório de psiquiatria infantil do HEWA aos 6 anos e 9 meses após ser encaminhado pelo Núcleo de Atendimento Neuropsiquiátrico Infantil (NANI) da UNIFESP por queixas de comportamento agitado, desatenção e dificuldade de aprendizagem. Foi realizada avaliação clínica, fundamentada no DSM-4, preenchendo os critérios para o diagnóstico de TDAH tipo combinado e transtorno desafiador opositor (TOD).

Em sua primeira avaliação ambulatorial, mãe relatou que criança nasceu a termo sem intercorrências na gestação. Em relação ao desenvolvimento neuropsicomotor, houve um atraso na sustentação do polo cefálico que ocorreu após os 6 meses de idade, sem atrasos em outros marcos do desenvolvimento. Mãe relata independência nas atividades diárias. Pais negaram antecedentes familiares de doenças psiquiátricas, apenas referindo que os dois irmãos mais velhos necessitaram de acompanhamento com fonoaudiólogo devido dificuldades de aprendizagem, sem outras implicações.

Desde o início da vida escolar, aos 6 anos, o indivíduo não apresentava boa adaptação, boa relação com colegas e professores. Em casa, também, não possui boa interação com familiares, ficando incomodado com a 
presença de visitas. Além disso, mãe relatou atitudes impulsivas e um comportamento desafiador quando contrariado, independentemente do local. Também relatou compulsividade e ansiedade em lugares desconhecidos, como aniversários.

Em 2011, aos 6 anos de idade, apresentava comportamento agitado, relatado desde os 2 anos, e dificuldade de aprendizado. Mãe relatou que no ano anterior paciente começou tratamento com neurologista, iniciando uso de Metilfenidato (10 mg) e Risperidona $(0,5 \mathrm{mg})$.

Ainda em 2011, durante acompanhamento no NANI, paciente foi submetido a uma avaliação interdisciplinar focada em uma abordagem neuropsicológica que verificou presença de sintomas como ansiedade, déficit de atenção, hiperatividade e problema desafiante opositor. Além disso, demonstrou capacidade intelectual acima da média, inadequação nos aspectos sociais e discursos fantasiosos. Na avaliação psicopedagógica demonstrou rendimento abaixo do esperado para a idade.

Após início da medicação, começou a apresentar melhora significativa no desempenho escolar, da atenção e da agitação.

Em 2012, mãe relata que a dificuldade no aprendizado melhorou, porém continua com dificuldade em escrever e fazer contas. Relata episódios de comportamento agressivo quando repreendido pela mãe, ofendendo-a verbalmente, contudo não há queixas de mal comportamento na escola. Assim, optou-se pelo aumento da dosagem de Risperidona noturno (0-1$1,5)$

Houve um episódio no qual o paciente ficou sem ambas as medicações, tendo piorado na agitação e presença de pesadelos. Nesse mesmo período, pai foi internado devido alcoolismo, fator que interfere desfavoravelmente no desenvolvimento emocional e cognitivo da criança. Com a volta da medicação, paciente apresentou melhora significativa, tendo alguns episódios de recaída, principalmente problemas de relacionamento na escola.

Em 2014, paciente mudou de escola, professora refere que fez amigos e está mais atento, porém apresenta comportamento agressivo quando recebe ordens. 
Em 2015, ficou um mês sem medicações, retornando ao estado agitado, desatento, além da dificuldade em manter o sono. Durante consulta, encontrava-se agitado e desafiador.

No ano seguinte, com 11 anos, aprendeu a ler e escrever, porém durante consulta, recusava-se a fazê-los, dizendo "é chato". Apresentou comportamento inadequado. Foi orientado à mãe a importância de impor limites. Paciente também relata episódios de discussão verbal com professora e colegas da escola. Decidiu-se aumentar as medicações, o que o deixou mais calmo.

Em 2018, na sexta série, mudou-se para escola pública. Relata que está sofrendo bullying por conta da altura e atraso escolar. Neste período foi orientada a redução da Risperidona, com posterior desmame. Paciente relatou que se sente bem e atento. Porém, houve queda do rendimento em algumas matérias e piora da memória. Decidiu-se pelo aumento do Metilfenidato. Além das queixas comportamentais, relatou que seu colega de classe lhe ofereceu drogas, mas recusou. Segundo paciente, repetidos episódios de ofensas de seus colegas o fizeram brigar. Para o controle da irritabilidade aumentou-se a Risperidona.

Em 2019, aos 15 anos, mãe relata que filho passa o dia jogando videogame e tenta "reviver" o jogo e seus personagens. Refere também que ele brinca com crianças de 6 anos e quando tenta fazer amizade com pessoas da mesma faixa etária, tem dificuldade.

No final de 2019, paciente ainda apresentava baixo desempenho escolar, não querendo ir a aula. Mãe relatou que as vezes seu comportamento continuava agitado. Paciente demonstra ter consciência de suas dificuldades e refere que o uso de Metilfenidato o ajuda a organizar melhor seu raciocínio.

\section{Discussão}

A partir do relato e baseado nos critérios do DSM-5 podemos realizar o diagnóstico de TDAH. Desde o início, foi identificado sintomas desse transtorno no paciente, como dificuldade em manter a atenção na escola, na realização de atividades e ao se envolver em tarefas que exijam esforço mental prolongado. Após longo acompanhamento, o TB passou a ser uma hipótese diagnóstica, que posteriormente foi confirmada devido à 
instabilidade emocional caracterizada por irritabilidade, agressividade flutuante e ideação delirante.

O diagnóstico do TB é relativamente novo na psiquiatria infantil, essa patologia apresenta diversos aspectos em comum com o TDAH, como epidemiologia, comorbidade e características clínicas. O TB é raro na infância, porém sua comorbidade com TDAH é alta. Nesse caso, é possível observar que o paciente apresenta TDAH de apresentação combinada e estudos mostram que a apresentação combinada foi significativamente maior do que a apresentação desatenta em crianças com TDAH e TB comórbido, comparadas às crianças sem TB. [7]

Em relação aos sintomas, o TDAH compartilha características importantes comuns ao TB na infância, presentes na Tabela 1.

Tabela 1. Sintomas comuns ao TDAH e Bipolares. [ㅁ] $(+)=$ presente

\begin{tabular}{|c|c|c|c|}
\hline & TB & TDAH & TB/TDAH \\
\hline Distração & & & + \\
\hline Loquacidade excessiva & & & + \\
\hline Perda de inibição social & & & + \\
\hline Mudanças de humor & & & + \\
\hline $\begin{array}{l}\text { Humor elevado/ atitude } \\
\text { grandiosa }\end{array}$ & + & & \\
\hline $\begin{array}{l}\text { Hiperatividade/ } \\
\text { Ansiedade }\end{array}$ & $\begin{array}{l}\text { Períodos } \\
\text { intermitentes, } \\
\text { caracterizados por } \\
\text { impulsividade e } \\
\text { agressividade }\end{array}$ & $\begin{array}{l}\text { Caracterizado por } \\
\text { hiperatividade } \\
\text { motora, inquietude } \\
\text { e relacionado } \\
\text { normalmente a } \\
\text { atividades que } \\
\text { exijam } \\
\text { concentração }\end{array}$ & \\
\hline Hipersexualidade & + & & \\
\hline $\begin{array}{l}\text { Distúrbio de } \\
\text { aprendizagem }\end{array}$ & $\begin{array}{l}\text { Desempenho } \\
\text { escolar } \\
\text { normalmente varia } \\
\text { conforme as fases } \\
\text { da doença }\end{array}$ & $\begin{array}{l}\text { Relacionado a } \\
\text { atividade } \\
\text { intelectuais } \\
\text { prolongadas. } \\
\text { Desempenho } \\
\text { escolar mais } \\
\text { homogêneo }\end{array}$ & \\
\hline $\begin{array}{l}\text { Diminuição da } \\
\text { necessidade de sono }\end{array}$ & + & & \\
\hline
\end{tabular}

A distração, apesar de ser um dos sintomas mais característicos desses dois transtornos, tem pouca utilidade no diagnóstico de patologias em crianças por ser comum em diversos transtornos com início na infância. [ㅁ] 
Durante crises maníacas, crianças e adolescentes podem apresentar, também, sintomas psicóticos, sendo os mais relatados foram alucinações visuais, auditivas, delírios de perseguição e grandeza. O paciente busca nas suas fantasias refugo de certas situações que causam desconforto em sua vida. Como consequência, apresenta desenvolvimento emocional prejudicado, intolerância à frustração e incapacidade de controlar seu comportamento muitas vezes inadequado, impactando negativamente em seu relacionamento interpessoal. [4]

As dificuldades de aprendizagem podem ser provenientes de problemas relacionados à escola, família e questões emocionais e psicólogas. A oscilação do humor do portador de TB gera danos ao desenvolvimento cognitivo, emocional e social da criança. [11]

Está bem estabelecido que várias funções cognitivas estão prejudicadas em crianças com diagnóstico de bipolaridade. Estudos neuropsicológicos recentes destacaram a natureza generalizada e severa dos prejuízos cognitivos nas fases dos episódios agudos. Esses déficits podem persistir mesmo durante os estados eutímicos. Evidências indicam que crianças com TBP apresentam diversos déficits neuropsicológicos, com desempenho significativamente pior nas funções executivas, como memória de trabalho auditiva e visuoespacial, flexibilidade cognitiva e tarefas de aprendizagem verbal. Com relação ao TDAH, pesquisas neuropsicológicas sugeriram prejuízos na velocidade de processamento, velocidade motora e memória declarativa verbal e em funções executivas, como memória de trabalho e set-shifting. [ㅁ]

Pesquisas atuais demonstraram a necessidade de buscar marcadores não sintomáticos, como perfis neuropsicológicos e neuroimagem para auxiliar no diagnostico desses transtornos. Diferentemente dos sintomas, estudos recentes indicaram distinção entre déficits neurocongnitivos entre o TDAH e o TB. O TDAH possui disfunções nos domínios da memória de trabalho verbal, espacial e na fluência verbal fonêmica. Enquanto o TB possui vários déficits, como atenção sustentada, memória de trabalho e verbal, flexibilidade verbal e cognitiva, presentes em todas as idades e durante todas as fases bipolares, maníacas e depressivas, persistindo na fase eutímica. []]

A recomendação clínica atual para o tratamento da comorbidade é iniciar a estabilização do humor, usou-se Risperidona $0,5 \mathrm{mg}$ pela manhã, e com a estabilização e se houver persistência de sintomas do TDAH, associar 
metilfenidato (10 mg pela manhã). Estudos adicionais são necessários para elucidar definitivamente o papel dos psicoestimulantes em pacientes com comorbidade. [10]

\section{Conclusão}

A comorbidade entre TDAH e TB costuma ocorrer e é comumente associada a outras condições médicas e psiquiátricas, comprometendo o desenvolvimento normal de quase todos os âmbitos da vida da criança e adolescente, principalmente no social e educacional, provocando grande sofrimento.

Por esses transtornos terem evolução crônica, compartilharem sintomas comuns e, frequentemente, se manifestarem em comorbidade, seu diagnóstico diferencial se torna difícil e ao mesmo tempo fundamental. Além disso, o TB pode piorar o prognóstico e dificultar o tratamento das crianças com TDAH, pois seu quadro clínico e os medicamentos usados para seu tratamento podem cursar com piora da atenção, da inquietação e da impulsividade.

É necessário a realização de novas pesquisas e debates voltados a esse tema, principalmente com enfoque em aspectos que não se sobreponham a fim de facilitar o diagnóstico comórbido e/ou diferencial do TB e TDAH na infância e consequentemente, minimizar seus prejuízos ao indivíduo. 


\section{Referências}

1. Marangoni C, De Chiara L, Faedda GL. Bipolar disorder and ADHD: comorbidity and diagnostic distinctions. Curr Psychiatry Rep. 2015 Aug;17(8):604. doi: 10.1007/s11920-015-0604-y. PMID: 26084666. https://doi.org/10.1007/s11920-015-0604-y PMid:26084666

2. Donfrancesco R, Di Trani M, Andriola E, et al. Bipolar Disorder in Children With ADHD: A Clinical Sample Study. J Atten Disord. 2017;21(9):715-720. https://doi.org/10.1177/1087054714539999 PMid:25015582

3. AMERICAN PSYCHIATRIC ASSOCIATION. Manual diagnóstico e estatístico de transtornos mentais: DSM-5. 5.ed. Porto Alegre: Artmed, 2014.

4. FU-I, Lee. Transtorno afetivo bipolar na infância e na adolescência. [S. I.], 18 out. 2006. Disponível em:

https://www.scielo.br/j/rbp/a/GQ8NxfmrVfdBhyH5W3P7GBc/?lang= pt Acesso em: 18 jul. 2021.

5. Moraes, Renata Garcia de Almeida, Gon, Márcia Cristina Caserta, \& Zazula, Robson. (2016). Transtorno bipolar em crianças e adolescentes: critérios para diagnóstico e revisão de intervenções psicossociais. Psico, 47(1), 77-87. https://doi.org/10.15448/1980$\underline{8623.2016 .1 .19994}$

6. GOLDMAN, Lee; AUSIELLO, Dennis. Cecil Medicina Interna. 25. ed. Saunders-Elsevier, 2018.

7. Naguy A. (2018). ADHD-juvenile bipolar disorder: mimics and chameleons!. World journal of pediatrics : WJP, 14(6), 525-527. https://doi.org/10.1007/s12519-018-0124-z - PMid:29446039

8. Zaravinos-Tsakos F, Kolaitis G. Disentangling pediatric bipolar disorder and attention deficit-hyperactivity disorder: A neuropsychological approach. Psychiatriki. 2020 Oct-Dec;31(4):332340. https://doi.org/10.22365/jpsych.2020.314.332 PMid:33361063 
9. Cichón, Lena; Jana- Kozik, Siwiec, Andrzej; Rybakowski, Janusz. Clinical picture and treatment of bipolar affective disorder in children and adolescents. Psychiatr. Pol. 2020; 54(1): 35-50. https://doi.org/10.12740/PP/OnlineFirst/92740 - PMid:32447355

10. Moraes, César de, Silva, Fábio Mello Barbirato Nascimento, \& Andrade, Ênio Roberto de. (2007). Diagnóstico e tratamento de transtorno bipolar e TDAH na infância: desafios na prática clínica. Jornal Brasileiro de Psiquiatria, 56(Suppl. 1), 19-24. https://doi.org/10.1590/S0047-20852007000500005

11. BRUM, Lanúzia Almeida; ZENI, Cristian Patrick; TRAMONTINA, Silzá. Aprendizagem e transtorno bipolar: reflexões psicopedagógicas. Rev. psicopedag., São Paulo , v. 28, n. 86, p. 194-200, 2011. Disponível em:

http://pepsic.bvsalud.org/scielo.php?script=sci arttext\&pid=S0103$84862011000200010 \& \operatorname{lng}=$ pt\&nrm $=$ iso . 
Daniel Cardoso de Bastos

$\underline{\text { ORCID Lattes }}$

Lorena Lourenço da Cruz de Carvalho

$\underline{\text { ORCID Lattes }}$

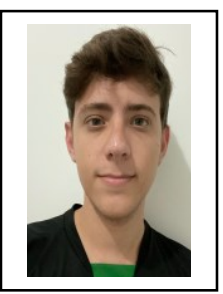

Vitor Melo Zibordi

$\underline{\text { ORCID Lattes }}$

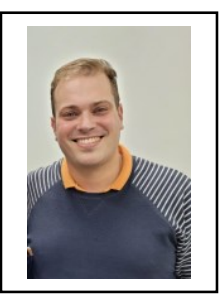

Higor Bermudes Nascimento

$\underline{\text { ORCID }}$

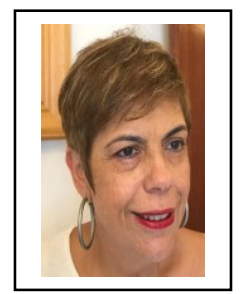

Sonia Maria Motta Palma

$\underline{\text { ORCID Lattes }}$ 